\title{
The Learning Curve for Laparoscopic Totally Extraperitoneal Herniorrhaphy by Logarithmic Function
}

\author{
Oh Chul Kwon, M.D., Yong Hae Baik, M.D., Ph.D., Min Gu Oh, M.D., Ph.D., Yeong Jin Park, M.D., Ph.D., Beom Seok Kwak, M.D.,
} Ph.D., In Woong Han, M.D., M.S.

Department of Surgery, Dongguk University Ilsan Hospital, Goyang, Korea

Purpose: Totally extraperitoneal (TEP) hernia repair has gained in popularity in the past two decades. Despite the advantages TEP hernia repair, the approach is hindered by the relatively long learning curve of the surgery. We tried to estimate the necessary number of repetitions of TEP hernia repair in the learning curve using logarithmic and exponential function models.

Methods: We performed a retrospective review of all patients who underwent TEP hernia repair by a single surgeon consecutively at a single center. We calculated how many operations were needed to achieve a reduction in the expected operating time to mean operating time using logarithmic and exponential function models.

Results: In the 91 patients, the logarithmic function model predicted that 37 cases were needed to overcome the learning curve for TEP hernia repair while the exponential model predicted that 39 cases were needed.

Conclusion: According to this study, at least 37 to 39 cases are needed in the overcome learning curve of TEP hernia repair. Further studies are needed to optimize surgical education and maximize quality.

Keywords: Herniorrhaphy, Learning curve, Likelihood functions

This is an Open Access article distributed under the terms of the Creative Commons Attribution Non-Commercial License (http:// creativecommons.org/licenses/by-nc/4.0/) which permits unrestricted non-commercial use, distribution, and reproduction in any medium, provided the original work is properly cited.
Received March 4, 2016

Revised September 1, 2016

Accepted September 1, 2016

Corresponding author

Yong Hae Baik

Department of Surgery, Dongguk

University Ilsan Hospital, Siksa-

dong, Ilsandong-gu, Goyang 10326,

Korea

Tel: +82-31-961-7269

Fax: +82-31-961-7144

E-mail: whitedrag@naver.com
Copyright (c) 2016 The Journal of Minimally Invasive Surgery. All rights reserved.

\section{INTRODUCTION}

Hernia repair is one of the most frequently performed operations in general surgery. The several Hernia repair techniques that have been devised - Lichtenstein's tension-free technique and laparoscopy for hernia repair - are landmarks that have allowed low postoperative pain, low recurrence rate and early return to normal activities.'

There are two laparoscopic approaches. Transabdominal preperitoneal (TAPP) hernia repair was developed first, and became the standard laparoscopic hernia repair technique with a recurrence rates under $1 \%$. But, the technique requires entry into the abdominal cavity and accesses the posterior floor of the groin, which can produce complications including internal hernias from inappropriate closure of the peritoneum and injury to the viscera from penetration of trocars and needles. ${ }^{2}$

Totally extraperitoneal (TEP) hernia repair was introduced in 1992. ${ }^{1}$ Since this technique does not require entry into the abdominal cavity and it is free from complications related to abdominal cavity entry, it has gained popularity in the recent two decades. ${ }^{3}$ Advantages of TEP hernia repair include equivalent recurrence rates as the open technique with less neural pain, less hematoma occurrence, lower infection rate and short recovery period. Compared to TAPP hernia repair, 


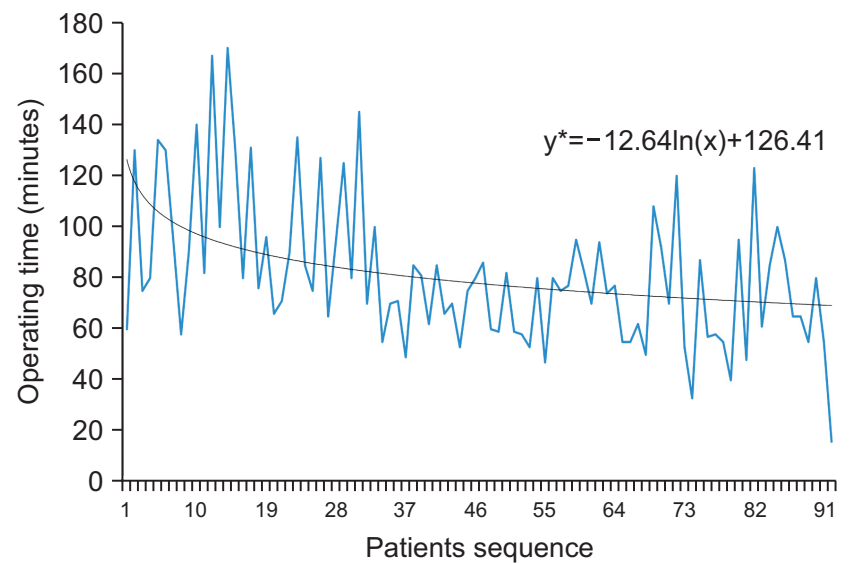

Fig. 1. The logarithmic model and formula. ${ }^{*} 81=$ Mean operation time (minutes).

TEP hernia repair has a shorter operation time and less possibility of damaging adjacent organs. ${ }^{4,5}$ Although postoperative complication rates are higher in the TAPP approach, most can be addressed by conservative management. Reoperation rate are similar in both approaches. ${ }^{6}$ A growing view among general surgeons is that both laparoscopic approaches are acceptable, with the chosen technique reflecting the clinical circumstances. In incarcerated hernia cases, for example, the TAPP hernia repair is usually preferred because it permits more accurate analysis of the contents of the hernia sac and of the viability, as well as safely reducing hernia contents. ${ }^{2}$ This review considers TEP hernia repair.

Despite the many advantages of TEP hernia repair, the relatively long learning curve needed to acquire sufficient skill at the laparoscopic approach is an important challenge. This laparoscopic approach is not an easy technique for beginner surgeons because of the complexity of inguinal anatomy and narrow space of the operation field. The clinical outcomes in the early cases can be compromised, making mastery of the technique as soon as possible important. The nature of learning curve, the exact learning model and the number of repetitions needed to become sufficiently skilled at the technique are still unclear. ${ }^{3}$

Assessment during the learning phase can include operating time, postoperative complications, and technical difficulties. ${ }^{8,9}$ In this study, only the operating time was used as a parameter since it is an objective parameter that is easily measured and which can be readily applied to produce mathematical models.

The aim of this study is to estimate the learning curve of TEP hernia repair using logarithmic and exponential function models.

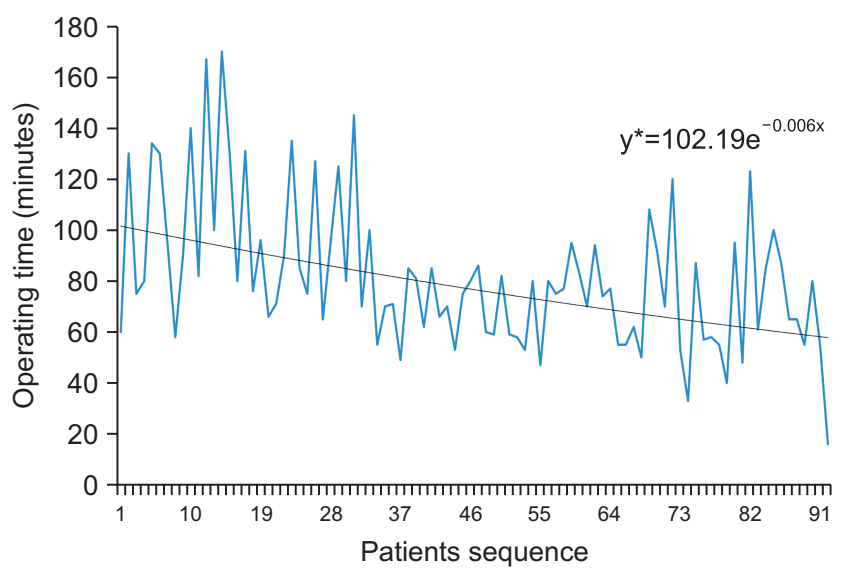

Fig. 2. The exponential model and formula. ${ }^{*} 81=$ Mean operation time (minutes).

\section{MATERIALS AND METHODS}

We performed a retrospective review of all patients who underwent TEP hernia repair by a single surgeon consecutively at a single center from September 2008 to May 2013. Clinical features, operating time, operative findings and outcomes were reviewed from medical records. A total of 101 patients underwent TEP hernia repair. Every operation was scheduled. Patients with both inguinal hernia or underwent other operation at same time were excluded. Finally, 91 patients were analyzed. A logarithmic function and an exponential function were derived from operating time data by univariate Cox regression analysis and graphs were also drawn with the number of operations plotted along the $\mathrm{X}$-axis and the operating time plotted along the Y-axis. We calculated how many operations were needed to achieve a reduction in the expected operating time to mean operating time (Fig. 1, 2). Statistical analysis was performed using SPSS version 18.0.

\section{Surgical technique}

Patients were instructed to empty their bladder before the operation. Under general anesthesia, all patients are placed in the supine position. The operator and the first assistant stood on opposite sides of the lesion. The main role of the first assistant was as a scopist. One $10 \mathrm{~mm}$ trocar and two $5 \mathrm{~mm}$ trocars were used. The $10 \mathrm{~mm}$ trocar was placed through a subumbilical incision. One $5 \mathrm{~mm}$ trocar was placed at the $\mathrm{su}^{-}$ prapubic area and another $5 \mathrm{~mm}$ trocar was placed between the other two trocars. The $10 \mathrm{~mm}$ balloon trocar proceeded along the anterior side of the posterior rectus sheath beyond the arcuate line. After inflation of the balloon catheter, $\mathrm{Coo}^{-}$ per's ligament, inferior epigastric vessel and hernia sac could 
be identified. In cases of indirect inguinal hernia, a hernia sac presented at the lateral side of the inferior epigastric vessel. Dissection of the medial side preceded dissection of the lateral side. Dissection of the lateral side began from lateral side of the inferior epigastric vessel. Securing of the pre-peritoneal space was attempted. The next step was careful dissection of the spermatic cord, since in cases of indirect hernia the hernia sac is encapsulated with the spermatic cord. In case of direct hernia, the hernia sac was usually separated and reduced by inflation of the balloon trocar. In this case, the pseudo sac was fixed to the Cooper's ligament to distinguish from relapse after surgery. After reduction of the hernia sac, artificial mesh (Parietex, $15 \times 10 \mathrm{~cm}$, Sofradium) was inserted between the abdominal wall and the peritoneum. The most important aspect in this procedure was peritonization of the hernia sac and parietalization of the spermatic cord. The medial margin of the mesh was fixed on the Cooper's ligament with a tack. After thrombin spraying and deflation with observing the inserted mesh on the proper position, surgery was completed.

\section{RESULTS}

Between September 2008 and May 2013, 101 patients underwent TEP hernia repair in our institute by single surgeon. Among these, 10 patients were excluded. Ninety one patients (84 males, seven females; mean age 50.0 years; mean BMI 23.8 $\mathrm{kg} / \mathrm{m}^{2}$ ) were finally included. There were 56 cases of rightsided hernia and 35 cases of left-sided hernia. There were 73 cases of indirect type, 12 cases of direct type and 6 cases of pantaloons type. Mean length of hospital stay was 2.92 days (range, 1 to 23 days). Hospitalization was exceptionally long in three cases -8 days in two cases and 23 days in one case. One

Table 1. Clinical parameters and operative outcomes

\begin{tabular}{lc}
\hline \multicolumn{1}{c}{ Parameter } \\
\hline Age (years) & $50.0 \pm 13.7(21.1 \sim 76.1)^{*}$ \\
Sex (male:female) & $85: 6^{\dagger}$ \\
$\mathrm{BMI}^{\S}\left(\mathrm{kg} / \mathrm{m}^{2}\right)$ & $23.8 \pm 2.6(18.1 \sim 31.9)^{*}$ \\
Location (right:left) & $56: 35^{\dagger}$ \\
Type of hernia (indirect:direct:pantaloon) & $73: 12: 6^{\dagger}$ \\
Operation time (minutes) & $81^{\ddagger}$ \\
Hospital stay (days) & $2.92^{\ddagger}$ \\
Early postoperative complication rate & $17.6 \%(16$ cases) \\
Recurrence rate & $2.2 \%(2$ cases) \\
${ }^{*}$ Mean \pm Standard deviation (range), ${ }^{\dagger}$ Number of patients, ${ }^{\ddagger}$ Mean, ${ }^{\S}$ Body \\
mass index.
\end{tabular}

patient who was hospitalized for 8 days desired a longer stay and the other requested treatment at the dermatology department for treatment of warts. The patient hospitalized for 23 days -was treated for incidentally found hepatocellular carcinoma after TEP hernia repair. Early postoperative complications included wound ecchymosis $(n=4)$, urinary retention $(n=3)$, subcutaneous emphysema $(n=2)$, scrotal swelling $(n=2)$, mild fever $(n=2)$, surgical site infection $(n=2)$ and wound hematoma $(n=1)$. All cases were minor (Clavien-Dindo grade I). There were no specific complications that required reoperation or additional hospital stay. The mean follow-up period was 31.8 months (range, 6.2 to 60.2 months). Recurrence occurred in two (2.2\%) patients (Table 1 ).

The logarithmic function and exponential function models demonstrating learning curve are depicted in Fig. 1 and 2, respectively. The mean operation time was 81 minutes. After the initial 37 cases were performed, the logarithmic function model predicted a decrease in operation time to less than the mean time. The exponential function model predicted that 39 cases would be necessary to reduce operation time to less than the mean time.

\section{DISCUSSION}

Inguinal hernia is one of the most common disease in general surgery, with over 700,000 cases of inguinal hernia repair performed annually in the United States and more than 33,000 cases performed annually in Korea. ${ }^{1,10}$ Inguinal hernia repair has an extensive history and refinements with time have lead to laparoscopic tension-free techniques that have proven very successful.

Despite this progress, laparoscopic hernia repair is not an easy technique for surgeons, especially for beginners, because of the anatomical complexity and the narrow operation field. Every surgeon has a period were skills are lacking and must be acquired. A learning curve that is as short as is safe and practical is important, because the early unskilled period is related to high complication and high recurrence rates.

The learning curve is a function that describing improvements of results that accrue from repetitive practice. The learning curve comprises three components: the starting point, slope of the curve, and plateau of the curve. ${ }^{11}$ The slope of the curve is a steep section between the starting point to the plateau. After the slope of the curve, a practitioner becomes stable and produces better results than before. In this study, we assumed the shape of learning curve followed either a logarithmic function or exponential function. The logarithmic regression model had the aforementioned shape, while the exponential model produced an almost linear shape.

The present clarification of the shape of the learning curve 
and the number of cases needed to acquire sufficient expertise in the procedure will help in the development of a training course. Beginner surgeon who receive little backup from an experienced surgeon can have more early recurrences due to lack of expertise. ${ }^{9}$ The approximate number of cases to overcome the early slope of the learning curve will help determine the length of time an experienced surgeon should oversee the learning phase, which should help to decrease the rate of complications during the early learning phase.

The logarithmic model and the exponential model predicted 36.3 and 38.7 cases, respectively, were needed to overcome the learning phase. The learning curve for TEP hernia repair was been extensively investigated. One study reported the use of a moving average method to determine that 30 cases were sufficient for development of expertise. ${ }^{8}$ Another study reported 60 cases were necessary. ${ }^{12}$ The European Hernia Society 2009 Guidelines suggest 50 to 100 cases are necessary, with the first 30 to 50 being critical. ${ }^{13}$ Fifty cases have been suggested in another study. Still, these numbers represent minimum estimates. Optimal outcomes and operative times may require up to 250 cases.

A formal training course, close intra-operative supervision and assistance provided to the surgeon by other well-trained staff may shorten the learning curve. ${ }^{14}$ Appropriate patient selection is an important parameter for successful surgery during the early period of learning. ${ }^{3}$ In the early period, TEP hernia repair may prudently avoid irreducible hernias and $\mathrm{pa}^{-}$ tients with previous lower quadrant surgery.

The weak point of this study is the small sample size and that surgeries were conducted by one surgeon. The may be the basis for the discrepancy in the determined number of cases and the markedly higher estimates in some other studies. Increasing case number may better reveal the shape of the late plateau. Also, more participating surgeons would clarify individual variations in the learning curves.

The mean operation time was 81 minutes. Compared to other literature it tend took longer. It is thought to be due to several time consuming cases during the early phase of the learning curve.

The strength of the study is the application of mathematical models. The application of mathematical models enables an objective derivation of the number of cases needed to gain adequate expertise.

According to this study, at least 37 to 39 cases are needed in the overcome learning curve of TEP hernia repair. Several ways to reduce the number of learning curve cases have been suggested in other studies. Clarification of the learning curve and the learning process of a surgeon would help in the development of training courses. Effective training courses are important for surgeons and patients.

\section{REFERENCES}

1) Dulucq JL, Wintringer P, Mahajna A. Laparoscopic totally extraperitoneal inguinal hernia repair: lessons learned from 3,100 hernia repairs over 15 years. Surg Endosc 2009;23:482-486.

2) Felix EL. Laparoscopic inguinal hernia repair. In: Swanström LL, Soper NJ, Leonard M, editors. Mastery of endoscopic and laparoscopic surgery. Fourth ed. Philadelphia: Wolters Kluwer/Lippincott Williams \& Wilkins Health; 2014. p.574-588.

3) Hasbahceci M, Basak F, Acar A, Alimoglu O. A New Proposal for Learning Curve of TEP Inguinal Hernia Repair: Ability to Complete Operation Endoscopically as a First Phase of Learning Curve. Minim Invasive Surg 2014;2014:528517.

4) Eklund AS, Montgomery AK, Rasmussen IC, Sandbue RP, Bergkvist LA, Rudberg CR. Low recurrence rate after laparoscopic (TEP) and open (Lichtenstein) inguinal hernia repair: a randomized, multicenter trial with 5-year follow-up. Ann Surg 2009;249:33-38.

5) Liem MS, van der Graaf Y, van Steensel CJ, et al. Comparison of conventional anterior surgery and laparoscopic surgery for inguinal-hernia repair. N Engl J Med 1997;336:1541-1547.

6) Kockerling F, Bittner R, Jacob DA, et al. TEP versus TAPP: comparison of the perioperative outcome in 17,587 patients with a primary unilateral inguinal hernia. Surg Endosc 2015;29:3750-3760.

7) Schouten N, Simmermacher RK, van Dalen T, et al. Is there an end of the "learning curve" of endoscopic totally extraperitoneal (TEP) hernia repair? Surg Endosc 2013;27:789-794.

8) Lim JW, Lee JY, Lee SE, et al. The learning curve for laparoscopic totally extraperitoneal herniorrhaphy by moving average. J Korean Surg Soc 2012;83:92-96.

9) Liem MS, van Steensel CJ, Boelhouwer RU, et al. The learning curve for totally extraperitoneal laparoscopic inguinal hernia repair. Am J Surg 1996;171:281-285.

10) National Health Insurance Corporation. Top 20 Frequent Diseases by Operation. Daejeon: National Health Insurance Corporation; 2011 [cited 2015 Sep 10]. Available from: http://kosis.kr/ statHtml/statHtml.do?orgId=350\&tblId=DT_35004_A07\&conn_ path=I2\&language=en.

11) Harrysson IJ, Cook J, Sirimanna P, Feldman LS, Darzi A, Aggarwal R. Systematic review of learning curves for minimally invasive abdominal surgery: a review of the methodology of data collection, depiction of outcomes, and statistical analysis. Ann Surg 2014;260:37-45.

12) Choi YY, Kim Z, Hur KY. Learning curve for laparoscopic totally extraperitoneal repair of inguinal hernia. Can J Surg 2012;55:33-36.

13) Simons MP, Aufenacker T, Bay-Nielsen M, et al. European Hernia Society guidelines on the treatment of inguinal hernia in adult patients. Hernia 2009;13:343-403.

14) Kim MC, Jung GJ, Kim HH. Learning curve of laparoscopy-assisted distal gastrectomy with systemic lymphadenectomy for early gastric cancer. World J Gastroenterol 2005;11:7508-7511. 